\title{
The Association Between Metabolic Syndrome and Heart Failure in Middle-aged Men and Women : Population-Based Study of 2 Million Individuals
}

\section{Tae-Eun Kim}

Konkuk University Seoul Hospital: Konkuk University Medical Center

Hyeongsu Kim

Konkuk University School of Medicine

JiDong Sung

Samsung Medical Center

Duk-Kyung Kim

Samsung Medical Center

Myoung-Soon Lee

Sungkyunkwan University School of Medicine

\section{Seong Woo Han}

Hallym University College of Medicine

Hyun-Joong Kim

Konkuk University Seoul Hospital: Konkuk University Medical Center

Sung Hea Kim ( $\square$ shkim@kuh.ac.kr)

Konkuk University Seoul Hospital: Konkuk University Medical Center https://orcid.org/0000-0001-58097958

\section{Kyu-Hyung Ryu}

Hallym University College of Medicine

\section{Original investigation}

Keywords: metabolic syndrome, heart failure, population-based study, gender difference

Posted Date: July 26th, 2021

DOl: https://doi.org/10.21203/rs.3.rs-719577/v1

License: (c) (1) This work is licensed under a Creative Commons Attribution 4.0 International License.

Read Full License 


\section{Abstract}

Background: Although the association between metabolic syndrome (MetS) and heart failure (HF) risk is known, large longitudinal studies that can permit causal inferences are limited. In this study, we investigated metabolic status as a risk factor for HF in middle-aged men and women and evaluated sex differences in various risk factors for $\mathrm{HF}$ using nationwide real-world data.

Methods: Data obtained from the Korean National Health Insurance Service from 2009 to 2016 were analyzed. A total of 2151597 middle-age subjects (1149642 men and 1001955 women) (between 50 and 59 years old) without a history of Cardiovascular or cerebrovascular disease or malignancy were enrolled. Subjects were divided into three groups according to the number of MetS components: 0 (normal), 1-2 (Pre-MetS) or 3-5 (MetS) based on the National Cholesterol Education Program Adult Treatment Panel III criteria. Cox proportional hazard models were used to estimate the association between MetS and incident HF after adjusting for clinical risk factors.

Results: At baseline, MetS existed in $23,77 \%$ of men and $10.58 \%$ of women. Pre-MetS and MetS increased the risk of HF: the hazard ratio (95\% confidence interval) of pre-MetS was 1.508 (1.287-1.767) in men and 1.395 (1.158-1.681) in women; the hazard ratio (95\% confidence interval) of MetS was 1.711 (1.4332.044) in men and 2.144 (1.674-2.747) in women. Current smoking, low hemoglobin level, underweight $(\mathrm{BMl}<18.5)$ and high creatinine level as well as acute myocardial infarction were also predictors of $\mathrm{HF}$ in both men and women.

Conclusions: This study confirmed that pre-MetS and MetS are risk factors for HF in middle-aged men and women. The effect of MetS on the occurrence of HF was stronger in women than in men. Pre-MetS was also a predictor of HF but was associated with a lower risk than MetS.

\section{Introduction}

Heart failure (HF) is a complex clinical syndrome characterized by a reduced ability to eject and/or accommodate blood within physiological pressure levels. ${ }^{1,2}$ Unlike other cardiac disorders, the prognoses of which have been markedly improved, only modest survival improvement has been observed in $\mathrm{HF}$ patients and the prevalence of $\mathrm{HF}$ is rising. ${ }^{3,4}$

Metabolic syndrome (MetS), a clustering of cardiovascular risk factors that includes hypertension, central obesity, insulin resistance, and atherogenic dyslipidemia, is common around the world. The prevalences of MetS in men and women are $35.6 \%$ and $30.3 \%$ in the United States ${ }^{5}$ and are $41 \%$ and $38 \%$ in Europe. ${ }^{6}$ Although the pathophysiologic mechanism of MetS has not been fully elucidated, obesity and insulin resistance are believed to play a critical role in the pathogenesis of MetS. ${ }^{7}$

Many studies have demonstrated the association between HF and MetS. Miura et al. ${ }^{8}$ reported that the prevalence of MetS is 2-fold higher in patients with HF than in the general population, and Li et al. ${ }^{9}$ found that MetS was associated with an approximately 2-fold increased likelihood of self-reported HF in a 
population-based cross-sectional study. Various studies have reported the association between MetS and the prognosis of HF. ${ }^{10,11}$ Although the underlying pathophysiologic mechanisms of the association between MetS and HF remain to be fully elucidated, metabolic stress caused by elevated glucose and free fatty acids, including dysregulated insulin signaling, impaired mitochondrial respiration and reactive oxygen species formation, is thought to decrease adenosine triphosphate (ATP) production, resulting in impaired contraction, myocellular hypertrophy, and fibrosis of the heart, subsequently leading to $\mathrm{HF}^{12}$

Despite the evidence showing the association between MetS and HF, longitudinal studies that can permit causal inferences are limited, and most of them were performed in elderly populations. Thus, in this study, we investigated MetS as a predictor of HF in middle-aged men and women using the National Health Insurance Service (NHIS) database. Additionally, we investigated the difference in the degree of the risk of MetS associated with HF between men and women.

\section{Methods}

\section{Database source}

This study used the NHIS database, which is the universal health insurance system in South Korea. The NHIS covers more than $97 \%$ of the population and contains information on patients' demographics (age, sex and socioeconomic variables, etc.), prescribed drugs (generic drug name, prescription date, duration and route of administration) and use of medical care institutions (hospital admissions, outpatient department visits, pharmaceutical visits, etc.). The NHIS also provides an annual or biennial health screening examination called the National Health Screening Program (NHSP) to the population aged 20 years and older, which includes questionnaires on lifestyle and behavior, physical examinations, and blood tests. In the NHIS database, diagnoses are coded according to the International Classification of Diseases-10th revision (ICD-10). The data were provided by NHIS after deidentification. This study was approved by the NHIS of Korea (No. NHIS-2020-1-153) and the Institutional Review Board of Konkuk University Medical Center (No. KUH 2020-07-096).

\section{Study population}

The number of individuals who underwent health screening examinations between January 2009 and December 2009 in South Korea was 9,927,538. Among the 9,927,538 participants, 7,775,941 were excluded for the following reasons: 1 ) $<50$ years old or $\geq 60$ years old, 2 ) history of malignancy (ICD-10 codes C00.X-C99.X) or 3) history of cardiovascular or cerebrovascular disease, including AF (ICD-10 codes 148), coronary artery disease (procedure codes M6561-4), myocardial infarction (ICD-10 codes I21), HF (ICD-10 codes I42 or I50), cerebrovascular accident (ICD-10 codes 160.X-1609. X), and peripheral arterial disease (ICD-10 codes 173 or 174). HF was diagnosed when the diagnostic code of HF occurred and there was any history of admission to hospital. The final number of participants in the study was $2,151,597$. These subjects were then divided into three groups for each sex according to the number of MetS components: normal group (0), pre-MetS group (1-2), and MetS group (3-5). We analyzed followup data until December 31, 2016 (Fig. 1). 


\section{Definition of metabolic syndrome}

According to the modified criteria of the National Cholesterol Education Program (NCEP) Adult Treatment Panel III (ATP III) criteria, ${ }^{13}$ a diagnosis of MetS was made when at least three of the following five components were present: (1) abdominal obesity (WC $\geq 90 \mathrm{~cm}$ for men, $\geq 85 \mathrm{~cm}$ for women); (2) elevated $\mathrm{BP}$ (systolic $\mathrm{BP} \geq 130 \mathrm{mmHg}$ or diastolic $\mathrm{BP} \geq 85 \mathrm{mmHg}$ or treatment of previously diagnosed HTN); (3) elevated fasting glucose ( $\geq 100 \mathrm{mg} / \mathrm{dL}$ or treatment of previously diagnosed $\mathrm{DM})$; (4) high triglycerides (TGs) ( $\geq 150 \mathrm{mg} / \mathrm{dL}$ or drug treatment for high TGs); and (5) low high-density lipoprotein cholesterol (HDL-C) ( $<40 \mathrm{mg} / \mathrm{dL}$ for men, $<50 \mathrm{mg} / \mathrm{dL}$ for women or drug treatment for low HDL-C). Subjects with one or two MetS components were defined as having pre-MetS, and those with no MetS components were defined as normal.

\section{Primary outcome and follow-up}

The primary outcome of this analysis was the incidence of HF during follow-up period. We defined an HF event using newly occurring ICD-10 codes for HF (I50). Follow-up was initiated at the date of the health screening examination and ended at the incidence of HF, death, or 31 December 2016, whichever came first.

\section{Statistical analysis}

Descriptive statistics were used to present the characteristics of the study subjects. The comparisons of baseline characteristics among metabolic syndrome were performed by $\mathrm{X}^{2}$-test.

Cox proportional hazard models were used to estimate hazard ratios (HRs) and 95\% confidence intervals (Cls) for the incidence of HF during the follow-up period. The models were initially unadjusted, and further adjustments were made for demographic characteristics (age, smoking status, and exercise status) (model 1). Model 2 was adjusted for the same covariates as model 1 plus family history of HTN, stroke and DM. Covariates were added to those of previous models step by step; thus, model 3 was adjusted for body mass index (BMI) and laboratory results (hemoglobin, creatinine, total cholesterol, low-density lipoprotein cholesterol, and alanine aminotransferase (ALT)) as well as demographic characteristics and family history. In model 4, the final model was adjusted for demographic characteristics, family history, $\mathrm{BMI}$, laboratory results and occurrence of acute myocardial infarction (AMI) during follow-up period. All tests were two-sided, with a significance level of 0.05. All analyses were conducted using SAS version 9.4 (SAS Institute Inc., Cary, NC, USA).

\section{Results}

\section{Baseline characteristics}

A total of 1,149,642 men and 1,001,955 women were included in this analysis. At baseline, MetS existed in $23,77 \%$ of men and $10.58 \%$ of women. The prevalence of pre-MetS was $55.64 \%$ and $50.86 \%$ in men and women, respectively. Table 1 presents baseline characteristics by MetS status. The prevalence of 
MetS was significantly associated with smoking status, alcohol consumption and frequency of exercise. The prevalence of MetS also exhibited a significant association with family histories of HTN, DM and stroke; BMl; and clinical laboratory results of total cholesterol, hemoglobin, creatinine and ALT (Table 1) in both sexes. 
Table 1

Baseline characteristics of study population according to metabolic syndrome status

Male

$\begin{array}{lll}\text { Normal } & \begin{array}{l}\text { Pre- } \\ \text { MetS }\end{array} & \text { MetS } \\ \mathrm{N}= & \mathrm{N}= \\ 236669 & \mathrm{~N}= & 273269 \\ (20.59) & 639704 & (23.77) \\ & (55.64) & \end{array}$

Female

\begin{tabular}{|c|c|c|c|}
\hline $\begin{array}{l}\mathrm{P} \text { - } \\
\text { value }\end{array}$ & $\begin{array}{l}\text { Normal } \\
N= \\
386,322 \\
(38.56)\end{array}$ & 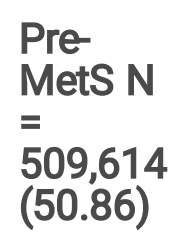 & $\begin{array}{l}\text { MetS } \\
N= \\
106,019 \\
(10.58)\end{array}$ \\
\hline
\end{tabular}

\section{Smoking \\ status}

$\begin{array}{lllllllll}\text { Non-smoker } & 69695 & 166871 & 63454 & < & 367335 & 483018 & 99076 & < \\ & (23.23) & (55.62) & (21.15) & 0.0001 & (38.69) & (50.87) & (10.44) & 0.0001 \\ \text { Ex-smoker } & 61530 & 172740 & 73774 & & 5641 & 6929 & 1642 & \\ & (19.97) & (56.08) & (23.95) & (39.69) & (48.75) & (11.55) & \\ \text { Current } & 103969 & 296719 & 134594 & & 10503 & 16075 & 4525 \\ \text { smoker } & (19.42) & (55.43) & (25.14) & & (33.77) & (51.68) & (14.55) & \end{array}$

Alcohol consumption

No drink

$\begin{array}{ll}76042 & 172399 \\ (24.28) & (55.04)\end{array}$

$64770<$

(20.68)

0.0001

270576

(38.33)

359005

(50.85)

$76363<$

2-3 per

114928

(20.92)

305899

128574

(23.4)

94636

(39.97)

119561

(50.50)

22545

$116225 \quad 57695$

1-4 per

32605

(15.79)

(56.28)

(27.94)

11672

(34.71)

17863

(53.13)

4089

$\geq 5$ per week

$\begin{array}{lll}10238 & 38795 & 19803 \\ (14.87) & (56.36) & (28.77)\end{array}$

3835

(33.26)

(52.89)

(12.16)

1597

(13.85)

\section{Exercise}

\begin{tabular}{|c|c|c|c|c|c|c|c|c|}
\hline No exercise & $\begin{array}{l}93741 \\
(20.12)\end{array}$ & $\begin{array}{l}257668 \\
(55.3)\end{array}$ & $\begin{array}{l}114561 \\
(24.59)\end{array}$ & $\hat{L}_{0.0001}$ & $\begin{array}{l}193655 \\
(37.68)\end{array}$ & $\begin{array}{l}263109 \\
(51.19)\end{array}$ & $\begin{array}{l}57202 \\
(11.13)\end{array}$ & $\stackrel{<}{0.0001}$ \\
\hline $\begin{array}{l}\text { 1-4 per } \\
\text { week }\end{array}$ & $\begin{array}{l}47105 \\
(20.44)\end{array}$ & $\begin{array}{l}127964 \\
(55.53)\end{array}$ & $\begin{array}{l}55388 \\
(24.03)\end{array}$ & & $\begin{array}{l}71043 \\
(39.03)\end{array}$ & $\begin{array}{l}92188 \\
(50.65)\end{array}$ & $\begin{array}{l}18771 \\
(10.31)\end{array}$ & \\
\hline$\geq 5$ per week & $\begin{array}{l}93704 \\
(21.11)\end{array}$ & $\begin{array}{l}248941 \\
(56.08)\end{array}$ & $\begin{array}{l}101220 \\
(22.8)\end{array}$ & & $\begin{array}{l}119010 \\
(39.76)\end{array}$ & $\begin{array}{l}150914 \\
(50.43)\end{array}$ & $\begin{array}{l}29360 \\
(9.81)\end{array}$ & \\
\hline
\end{tabular}

Unit: persons(\%)

Abbreviation: Pre-MetS, pre-metabolic syndrome; MetS, metabolic syndrome

P-value by $X^{2}$-test 


\section{Family \\ history of \\ hypertension}

$\begin{array}{lllllllll}\text { Yes } & 18324 & 65336 & 35905 & < & 40687 & 68343 & 18707 & < \\ & (15.33) & (54.64) & (30.03) & 0.0001 & (31.85) & (53.50) & (14.64) & 0.0001 \\ \text { No } & 145852 & 378540 & 156040 & & 213678 & 268648 & 53509 & \\ & (21.44) & (55.63) & (22.93) & & (39.88) & (50.14) & (9.99) & \end{array}$

\section{Family \\ history of \\ diabetes \\ mellitus}

$\begin{array}{lllllllll}\text { Yes } & 17843 & 59802 & 32617 & & 34730 & 52863 & 14922 & < \\ & (16.18) & (54.24) & (29.58) & 0.0001 & (33.88) & (51.57) & (14.56) & 0.0001 \\ \text { No } & 146165 & 383657 & 159236 & & 219524 & 283972 & 57220 & \\ & (21.21) & (55.68) & (23.11) & & (39.15) & (50.64) & (10.20) & \end{array}$

\section{Family \\ history of \\ stroke}

\begin{tabular}{llllllll} 
Yes & 16413 & 47273 & 21534 & 23950 & 33966 & 7533 & $<$ \\
& $(19.26)$ & $(55.47)$ & $(25.27)$ & $(36.59)$ & $(51.90)$ & $(11.51)$ & 0.0001 \\
\hline No & 47711 & 396629 & 170413 & 230167 & 302655 & 64531 & \\
& $(20.67)$ & $(55.49)$ & $(23.84)$ & $(38.53)$ & $(50.67)$ & $(10.80)$ &
\end{tabular}

\section{Total}

cholesterol

(mg/dL)

$\begin{array}{lllllllll}<200 & 151035 & 332175 & 119760 & < & 245784 & 301128 & 50829 & < \\ & (25.05) & (55.09) & (19.86) & 0.0001 & (41.12) & (50.38) & (8.5) & 0.0001 \\ 200-239 & 70069 & 226451 & 103697 & & 114051 & 156365 & 37389 & \\ & (17.51) & (56.58) & (25.91) & & (37.05) & (50.8) & (12.15) & \\ >239 & 15565 & 81078 & 49812 & & 26487 & 52121 & 17801 & \\ & (10.63) & (55.36) & (34.01) & & (27.47) & (54.06) & (18.46) & \end{array}$

\section{ALT (IU/L)}

$\begin{array}{lllllllll}<40 & 216861 & 526325 & 178156 & < & 376523 & 485460 & 91557 & < \\ & (23.54) & (57.13) & (19.34) & 0.0001 & (39.49) & (50.91) & (9.6) & 0.0001\end{array}$

Unit: persons(\%)

Abbreviation: Pre-MetS, pre-metabolic syndrome; MetS, metabolic syndrome

P-value by $\mathrm{X}^{2}$-test 


\begin{tabular}{|c|c|c|c|c|c|c|c|c|}
\hline & Male & & & & Female & & & \\
\hline $40-99$ & $\begin{array}{l}18121 \\
(8.6)\end{array}$ & $\begin{array}{l}105617 \\
(50.1)\end{array}$ & $\begin{array}{l}87087 \\
(41.31)\end{array}$ & & $\begin{array}{l}8696 \\
(19.87)\end{array}$ & $\begin{array}{l}21857 \\
(49.95)\end{array}$ & $\begin{array}{l}13209 \\
(30.18)\end{array}$ & \\
\hline$>100$ & $\begin{array}{l}1687 \\
(9.65)\end{array}$ & $\begin{array}{l}7762 \\
(44.42)\end{array}$ & $\begin{array}{l}8026 \\
(45.93)\end{array}$ & & $\begin{array}{l}1103 \\
(23.71)\end{array}$ & $\begin{array}{l}2297 \\
(49.37)\end{array}$ & $\begin{array}{l}1253 \\
(26.93)\end{array}$ & \\
\hline $\begin{array}{l}\text { Hemoglobin } \\
(\mathrm{g} / \mathrm{dL})\end{array}$ & & & & & & & & \\
\hline $\begin{array}{l}<13.5 \text { (Male), } \\
<12 \text { (Female) }\end{array}$ & $\begin{array}{l}23156 \\
(26.5)\end{array}$ & $\begin{array}{l}48700 \\
(55.74)\end{array}$ & $\begin{array}{l}15510 \\
(17.75)\end{array}$ & $\hat{L}_{0.0001}$ & $\begin{array}{l}90985 \\
(40.86)\end{array}$ & $\begin{array}{l}112912 \\
(50.7)\end{array}$ & $\begin{array}{l}18792 \\
(8.44)\end{array}$ & $<.0001$ \\
\hline $\begin{array}{l}13.5- \\
17.5(\mathrm{Male}) \\
12-15.5 \\
\text { (Female) }\end{array}$ & $\begin{array}{l}212536 \\
(20.25)\end{array}$ & $\begin{array}{l}584907 \\
(55.73)\end{array}$ & $\begin{array}{l}252155 \\
(24.02)\end{array}$ & & $\begin{array}{l}13166 \\
(37.99)\end{array}$ & $\begin{array}{l}394040 \\
(50.89)\end{array}$ & $\begin{array}{l}86041 \\
(11.11)\end{array}$ & \\
\hline $\begin{array}{l}>17.5 \text { (Male), } \\
> \\
15.5(\text { Female })\end{array}$ & $\begin{array}{l}977 \\
(7.71)\end{array}$ & $\begin{array}{l}6097 \\
(48.09)\end{array}$ & $\begin{array}{l}5604 \\
(44.2)\end{array}$ & & $\begin{array}{l}1151 \\
(23.3)\end{array}$ & $\begin{array}{l}2619 \\
(53.02)\end{array}$ & $\begin{array}{l}1170 \\
(23.68)\end{array}$ & \\
\hline $\begin{array}{l}\text { Creatinine } \\
\text { (mg/dL) }\end{array}$ & & & & & & & & \\
\hline$\leq 1.5$ & $\begin{array}{l}228987 \\
(20.67)\end{array}$ & $\begin{array}{l}616190 \\
(55.63)\end{array}$ & $\begin{array}{l}262468 \\
(23.70)\end{array}$ & $\begin{array}{l}< \\
0.0001\end{array}$ & $\begin{array}{l}381018 \\
(38.59)\end{array}$ & $\begin{array}{l}501914 \\
(50.84)\end{array}$ & $\begin{array}{l}104342 \\
(10.57)\end{array}$ & $\begin{array}{l}< \\
0.0001\end{array}$ \\
\hline$>1.5$ & $\begin{array}{l}7660 \\
(18.27)\end{array}$ & $\begin{array}{l}23478 \\
(56.00)\end{array}$ & $\begin{array}{l}10788 \\
(25.73)\end{array}$ & & $\begin{array}{l}5281 \\
(36.11)\end{array}$ & $\begin{array}{l}7671 \\
(52.45)\end{array}$ & $\begin{array}{l}1674 \\
(11.45)\end{array}$ & \\
\hline $\begin{array}{l}\text { Body Mass } \\
\text { Index } \\
\left(\mathrm{kg} / \mathrm{m}^{2}\right)\end{array}$ & & & & & & & & \\
\hline$<18.5$ & $\begin{array}{l}8184 \\
(48.01)\end{array}$ & $\begin{array}{l}8264 \\
(48.48)\end{array}$ & $\begin{array}{l}599 \\
(3.51)\end{array}$ & $\begin{array}{l}< \\
0.0001\end{array}$ & $\begin{array}{l}15574 \\
(61.25)\end{array}$ & $\begin{array}{l}9535 \\
(37.50)\end{array}$ & $\begin{array}{l}319 \\
(1.25)\end{array}$ & $\begin{array}{l}< \\
0.0001\end{array}$ \\
\hline $18.5-22.9$ & $\begin{array}{l}123762 \\
(34.53)\end{array}$ & $\begin{array}{l}205799 \\
(57.42)\end{array}$ & $\begin{array}{l}28877 \\
(8.06)\end{array}$ & & $\begin{array}{l}243305 \\
(49.84)\end{array}$ & $\begin{array}{l}227833 \\
(46.67)\end{array}$ & $\begin{array}{l}17019 \\
(3.49)\end{array}$ & \\
\hline 23-24.9 & $\begin{array}{l}68418 \\
(21.00)\end{array}$ & $\begin{array}{l}201763 \\
(61.92)\end{array}$ & $\begin{array}{l}55641 \\
(17.08)\end{array}$ & & $\begin{array}{l}85685 \\
(35.64)\end{array}$ & $\begin{array}{l}133579 \\
(55.57)\end{array}$ & $\begin{array}{l}21128 \\
(8.79)\end{array}$ & \\
\hline $25-29.9$ & $\begin{array}{l}36122 \\
(8.74)\end{array}$ & $\begin{array}{l}214726 \\
(51.95)\end{array}$ & $\begin{array}{l}162486 \\
(39.31)\end{array}$ & & $\begin{array}{l}41084 \\
(18.76)\end{array}$ & $\begin{array}{l}126189 \\
(57.63)\end{array}$ & $\begin{array}{l}51685 \\
(23.60)\end{array}$ & \\
\hline$>=30$ & $\begin{array}{l}183 \\
(0.52)\end{array}$ & $\begin{array}{l}9152 \\
(26.15)\end{array}$ & $\begin{array}{l}25666 \\
(73.33)\end{array}$ & & $\begin{array}{l}674 \\
(2.32)\end{array}$ & $\begin{array}{l}12478 \\
(43.00)\end{array}$ & $\begin{array}{l}15868 \\
(54.68)\end{array}$ & \\
\hline Unit: persons & & & & & & & & \\
\hline Abbreviation: & e-MetS, p & -metaboli & syndrome & ints & abolic syr & rome & & \\
\hline P-value by $X^{2}$ & & & & & & & & \\
\hline
\end{tabular}




\section{Association between MetS status and HF}

The incidence rate of HF was associated with MetS status. The incidences increased as pre-MetS progressed to MetS and were higher in men than in women. The rates per 100,000 person-years in the normal population were 19.2 in men and 13.38 in women, and the rates in the pre-MetS and MetS populations were 35.88 and 55.05 , respectively, in men and 19.42 and 34.91 , respectively, in women (Fig. 2).

Table 2 compares the baseline characteristics of the population with HF and those without HF. Both in men and women, the frequency of HF was higher in populations with MetS than in the normal population. The pre-MetS population also showed a higher frequency of HF than the normal population, although the proportion was lower than that of the MetS group. 
Table 2

Baseline characteristics of study population according to heart failure

Male

non-HF
HF
Female

$\begin{array}{llll}\begin{array}{l}\mathrm{p}- \\ \text { value }\end{array} & \text { non-HF } & \text { HF } & \begin{array}{l}\mathrm{p}- \\ \text { value }\end{array}\end{array}$

\section{Metabolic}

status

\begin{tabular}{|c|c|c|c|c|c|c|}
\hline Normal & $236373(99.87)$ & $296(0.13)$ & $\begin{array}{l}< \\
0.0001\end{array}$ & $386025(99.92)$ & $297(0.08)$ & $<.0001$ \\
\hline Pre-MetS & 638221 (99.77) & $\begin{array}{l}1483 \\
(0.23)\end{array}$ & & $509001(99.88)$ & $612(0.12)$ & \\
\hline MetS & 272285 (99.64) & $984(0.36)$ & & 105769(99.76) & $250(0.24)$ & \\
\hline \multicolumn{7}{|l|}{$\begin{array}{l}\text { Smoking } \\
\text { status }\end{array}$} \\
\hline Non-smoker & $299458(99.81)$ & $562(0.19)$ & $\begin{array}{l}< \\
0.0001\end{array}$ & $\begin{array}{l}948372 \\
(99.89)\end{array}$ & $\begin{array}{l}1056 \\
(0.11)\end{array}$ & $\begin{array}{l}<.0001 \\
0.0\end{array}$ \\
\hline Ex-smoker & 307501 (99.82) & $543(0.18)$ & & 14191 (99.85) & $21(0.15)$ & \\
\hline $\begin{array}{l}\text { Current } \\
\text { smoker }\end{array}$ & 533637 (99.69) & $\begin{array}{l}1645 \\
(0.31)\end{array}$ & & 31028 (99.76) & $75(0.24)$ & \\
\hline
\end{tabular}

\section{Alcohol consumption}

No drink

$312309(99.71) \quad 902(0.29)$
$<\quad 705123$
$0.0001 \quad(99.88)$

2-3 per

month

$\begin{array}{ll}548255(99.79) & 1146 \\ & (0.21)\end{array}$

236485

(99.89)

1-4 per week $206048(99.77) \quad 477(0.23)$

$33585(99.88) \quad 39(0.12)$

$\geq 5$ per week $\quad 68622(99.69) \quad 214(0.31)$

$11510(99.83) \quad 20(0.17)$

\section{Exercise}

No exercise $\quad 464750(99.74) \quad 1220$

(0.26)

0.0005

513340
$(99.88)$

$626(0.12)$

0.0963

1-4 per week

$229928(99.77) \quad 529(0.23)$

181793

(99.89)

$209(0.11)$

Unit: persons(\%)

Abbreviation: Pre-MetS, pre-metabolic syndrome; MetS, metabolic syndrome; HF, heart failure

P-value by $X^{2}$-test 


\begin{tabular}{|c|c|c|c|c|c|c|}
\hline \multirow{2}{*}{$\geq 5$ per week } & \multicolumn{3}{|l|}{ Male } & \multicolumn{3}{|l|}{ Female } \\
\hline & 442872 (99.78) & $993(0.22)$ & & $\begin{array}{l}298969 \\
(99.90)\end{array}$ & $314(0.10)$ & \\
\hline \multicolumn{7}{|l|}{$\begin{array}{l}\text { Family history } \\
\text { of } \\
\text { hypertension }\end{array}$} \\
\hline Yes & 119251 (99.74) & $314(0.26)$ & 0.0308 & $\begin{array}{l}127569 \\
(99.87)\end{array}$ & $168(0.13)$ & 0.0124 \\
\hline No & 678868 (99.77) & $\begin{array}{l}1564 \\
(0.23)\end{array}$ & & $\begin{array}{l}535269 \\
(99.89)\end{array}$ & $566(0.11)$ & \\
\hline \multicolumn{7}{|l|}{$\begin{array}{l}\text { Family history } \\
\text { of diabetes } \\
\text { mellitus }\end{array}$} \\
\hline Yes & 109954 (99.72) & $308(0.28)$ & 0.0011 & $\begin{array}{l}102395 \\
(99.88)\end{array}$ & $120(0.12)$ & 0.4831 \\
\hline No & 687487 (99.77) & $\begin{array}{l}1571 \\
(0.23)\end{array}$ & & $\begin{array}{l}560104 \\
(99.89)\end{array}$ & $612(0.11)$ & \\
\hline \multicolumn{7}{|l|}{$\begin{array}{l}\text { Family history } \\
\text { of stroke }\end{array}$} \\
\hline Yes & 85003 (99.75) & $217(0.25)$ & 0.2137 & 65377 (99.89) & $72(0.11)$ & 0.9986 \\
\hline No & 713089 (99.77) & $\begin{array}{l}1664 \\
(0.23)\end{array}$ & & $\begin{array}{l}596696 \\
(99.89)\end{array}$ & $657(0.11)$ & \\
\hline \multicolumn{7}{|l|}{$\begin{array}{l}\text { Total } \\
\text { cholesterol } \\
\text { (mg/dL) }\end{array}$} \\
\hline$<200$ & 601702 (99.79) & $\begin{array}{l}1268 \\
(0.21)\end{array}$ & $<.0001$ & $\begin{array}{l}597109 \\
(99.89)\end{array}$ & $631(0.11)$ & 0.0002 \\
\hline $200-239$ & 399277 (99.77) & $940(0.23)$ & & $\begin{array}{l}307422 \\
(99.88)\end{array}$ & $383(0.12)$ & \\
\hline$>239$ & 145900 (99.62) & $555(0.38)$ & & 96264 (99.85) & $145(0.15)$ & \\
\hline \multicolumn{7}{|l|}{ ALT (IU/L) } \\
\hline$<40$ & 919272 (99.78) & $\begin{array}{l}2070 \\
(0.22)\end{array}$ & $\begin{array}{l}< \\
0.0001\end{array}$ & $\begin{array}{l}952491 \\
(99.91)\end{array}$ & $\begin{array}{l}1048 \\
(0.09)\end{array}$ & $\begin{array}{l}< \\
0.0001\end{array}$ \\
\hline 40-99 & 210218 (99.71) & 607 (0.29) & & 43668 (99.88) & $337(0.12)$ & \\
\hline \multicolumn{7}{|c|}{ Unit: persons(\%) } \\
\hline \multicolumn{7}{|c|}{ Abbreviation: Pre-MetS, pre-metabolic syndrome; MetS, metabolic syndrome; HF, heart failure } \\
\hline P-value by $X^{2}$ & & & & & & \\
\hline
\end{tabular}




\begin{tabular}{|c|c|c|c|c|c|c|}
\hline & Male & & & Female & & \\
\hline$>100$ & 17389 (99.51) & $86(0.49)$ & & 159829(99.86) & $231(0.14)$ & \\
\hline \multicolumn{7}{|l|}{$\begin{array}{l}\text { Hemoglobin } \\
(\mathrm{g} / \mathrm{dL})\end{array}$} \\
\hline $\begin{array}{l}<13.5 \text { (Male) } \\
<12 \text { (Female) }\end{array}$ & 87087 (99.68) & $279(0.32)$ & $\begin{array}{l}<.0001 \\
0.0\end{array}$ & $\begin{array}{l}222419 \\
(99.88)\end{array}$ & $270(0.12)$ & $\begin{array}{l}<.054 \\
0.054\end{array}$ \\
\hline $\begin{array}{l}\text { 13.5- } \\
17.5 \text { (Male), } \\
12-15.5 \\
\text { (Female) }\end{array}$ & $\begin{array}{l}1047180 \\
(99.77)\end{array}$ & $\begin{array}{l}2418 \\
(0.23)\end{array}$ & & $\begin{array}{l}773363 \\
(99.89)\end{array}$ & $878(0.11)$ & \\
\hline $\begin{array}{l}>17.5 \text { (Male), } \\
> \\
15.5 \text { (Female) }\end{array}$ & $12612(99.48)$ & $66(0.62)$ & & 4929 (99.78) & $11(0.22)$ & \\
\hline \multicolumn{7}{|l|}{$\begin{array}{l}\text { Creatinine } \\
(\mathrm{mg} / \mathrm{dL})\end{array}$} \\
\hline$\leq 1.5$ & 1105044(99.77) & 2601(0.23) & $<.0001$ & 986158(99.89) & $1115(0.11)$ & $<.0001$ \\
\hline$>1.5$ & 41764(99.61) & $162(0.39)$ & & 14582(99.70) & $44(0.30)$ & \\
\hline \multicolumn{7}{|l|}{$\begin{array}{l}\text { Body Mass } \\
\text { Index }\left(\mathrm{kg} / \mathrm{m}^{2}\right)\end{array}$} \\
\hline$<18.5$ & 16995(99.69) & $52(0.31)$ & $<0.0001$ & 25396(99.87) & $32(0.13)$ & $\begin{array}{l}< \\
0.0001\end{array}$ \\
\hline $18.5-22.9$ & $357675(99.79)$ & $763(0.21)$ & & 487723(99.91) & $433(0.09)$ & \\
\hline $23-24.9$ & $325142(99.79)$ & $680(0.21)$ & & 240123(99.89) & $269(0.11)$ & \\
\hline $25-29.9$ & 412217(99.73) & 1117(0.27) & & 218619(99.85) & $339(0.15)$ & \\
\hline$>=30$ & $34850(99.57)$ & $151(0.43)$ & & 28934(99.70) & $86(0.30)$ & \\
\hline \multicolumn{7}{|c|}{ Unit: persons(\%) } \\
\hline \multicolumn{7}{|c|}{ Abbreviation: Pre-MetS, pre-metabolic syndrome; MetS, metabolic syndrome; $\mathrm{HF}$, heart failure } \\
\hline P-value by $X^{2}$ & & & & & & \\
\hline
\end{tabular}

In addition to MetS status, there were statistically significant differences in baseline characteristics between populations with and without HF. Both in men and women, current smokers showed a higher proportion of HF than ex-smokers or nonsmokers. An occurrence of AMI during follow-up period is definitely related to a higher frequency of HF. In comparison to the normal BMI group, both the underweight and overweight (obese) groups showed higher HF frequency in both sexes. Laboratory findings including cholesterol, creatinine and ALT and family history of hypertension were also related to 
the frequency of HF in both sexes. The differences in HF frequency according to alcohol consumption, frequency of exercise and family history of DM and hemoglobin level were shown only in men (Table 2).

\section{Risk factors for $\mathrm{HF}$}

Multivariable Cox regression analysis was performed to evaluate risk factors for $\mathrm{HF}($ Table 3,4$)$. Without other covariates, the HRs of pre-MetS and MetS for HF were $1.867(95 \% \mathrm{Cl}), 1.599-2.18)$ and 3.016 (2.569-3.541), respectively, in men and 1.605 (1.34-1.923) and 3.349 (2.706-4.145), respectively, in women (model 1). Considering age; smoking; alcohol consumption; exercise; family histories of heart disease, HTN, DM and stroke; laboratory results; and occurrence of AMI during follow-up period as covariates, the occurrence of HF was still significantly higher in subjects with pre-MetS or MetS than in normal individuals, with higher HRs observed in MetS patients than pre-MetS patients. The risk of HF associated with pre-MetS was slightly higher in men than in women, whereas that associated with MetS was higher in women than in men (HR $(95 \% \mathrm{Cl})$ of pre-MetS: $1.508(1.287-1.767)$ in men and 1.395 $(1.158-1.681)$ in women; HR $(95 \% \mathrm{Cl})$ of MetS: 1.711 (1.433-2.044) in men and 2.144 (1.674-2.747) in women) (model 5) (Table 3, 4). 
Table 3

Risk of heart failure in male: Cox proportional hazard model.

\section{Non-Adjusted Multivariable HR $(95 \% \mathrm{Cl})$ \\ $\mathrm{HR}(95 \% \mathrm{Cl})$}

\begin{tabular}{llllll} 
Metabolic status & Model $\mathbf{1}$ & Model $\mathbf{2}$ & Model $\mathbf{3}$ & Model $\mathbf{4}$ & Model $\mathbf{5}$ \\
\hline Normal & 1 & 1 & 1 & 1 & 1 \\
\hline Pre-metabolic synd. & $1.867(1.599-$ & 1.846 & 1.839 & 1.778 & $1.508(1.287-$ \\
& $2.18)$ & $(1.581-$ & $(1.575-$ & $(1.517-$ & $1.767)$ \\
& & $2.156)$ & $2.148)$ & $2.083)$ & \\
Metabolic synd. & $3.016(2.569-$ & 2.93 & 2.901 & 2.584 & $1.711(1.433-$ \\
& $3.541)$ & $(2.494-$ & $(2.469-$ & $(2.167-$ & $2.044)$ \\
Age & & $3.442)$ & $3.41)$ & $3.081)$ & \\
& & 1.067 & 1.067 & 1.069 & $1.034(1.017-$ \\
& & $(1.05-$ & $(1.05-$ & $(1.051-$ & $1.051)$
\end{tabular}

\section{Smoking status}

Non-smokiner

0.994
$(0.858-$
$1.151)$

1

1

1

\section{Ex-smoker \\ Alcohol \\ consumption \\ Current smoker}

$\begin{array}{llll}1.8 & 1.787 & 1.772 & 1.19(1.051- \\ (1.592- & (1.58- & (1.567- & 1.348) \\ 2.035) & 2.02) & 2.005) & \end{array}$

\begin{tabular}{lllll} 
No drink & 1 & 1 & 1 & 1 \\
2-3 per month & 0.64 & 0.638 & 0.643 & $0.814(0.729-$ \\
& $(0.573-$ & $(0.571-$ & $(0.576-$ & $0.909)$ \\
& $0.714)$ & $0.712)$ & $0.718)$ & \\
\hline 1-4 per week & 0.666 & 0.665 & 0.674 & $0.975(0.848-$ \\
& $(0.58-$ & $(0.579-$ & $(0.587-$ & $1.12)$ \\
& $0.765)$ & $0.764)$ & $0.774)$ & \\
$\geq 5$ per week & 0.806 & 0.807 & 0.816 & $1.162(0.961-$ \\
& $(0.667-$ & $(0.668-$ & $(0.675-$ & $1.404)$ \\
& $0.974)$ & $0.975)$ & $0.986)$ &
\end{tabular}

\section{Exercise}

No exercise

1

0.982
$(0.848-$
$1.138)$
1.787
$(1.58-$
$2.02)$

0.971

(0.838-

1.125)

0.906 (0.7821.05) 
Non-Adjusted Multivariable $\mathrm{HR}(95 \% \mathrm{Cl})$

$\mathrm{HR}(95 \% \mathrm{Cl})$

$\begin{array}{lllll}1-4 \text { per week } & 0.936 & 0.933 & 0.928 & 0.927(0.817- \\ & (0.826- & (0.822- & (0.818- & 1.051) \\ & 1.062) & 1.058) & 1.052) & \\ \geq 5 \text { per week } & 0.964 & 0.956 & 0.954 & 0.985(0.888- \\ & (0.869- & (0.862- & (0.86- & 1.094) \\ & 1.069) & 1.06) & 1.059) & \end{array}$

\section{Family history of} heart disease

$\begin{array}{llll}\text { No } & 1 & 1 & 1 \\ \text { Yes } & 1.328 & 1.339 & 1.153(0.964- \\ & (1.108- & (1.117- & 1.38)\end{array}$

\section{Family history of} hypertension

$\begin{array}{llll}\text { No } & 1 & 1 & 1 \\ \text { Yes } & 1.033 & 1.042 & 0.991(0.869- \\ & (0.904- & (0.912- & 1.13) \\ & 1.18) & 1.189) & \end{array}$

\section{Family history of} diabetes mellitus

$\begin{array}{llll}\text { No } & 1 & 1 & 1 \\ \text { Yes } & 1.11 & 1.116 & 1.055(0.926- \\ & (0.972- & (0.978- & 1.203) \\ & & 1.267) & 1.274)\end{array}$

\section{Family history of} stroke

$\begin{array}{llll}\text { No } & 1 & 1 & 1 \\ \text { Yes } & 0.985 & 0.997 & 0.982(0.844- \\ & (0.845- & (0.855- & 1.143)\end{array}$

\section{Body mass index}

$<18.5$

$18.5-22.9$

$$
\begin{aligned}
& 1.629 \\
& (1.154- \\
& 2.298)
\end{aligned}
$$

$1.786(1.266-$ 2.52)

1 1

Abbreviation: Pre-MetS, pre-metabolic syndrome; MetS, metabolic syndrome, HR, hazard ratio *Occurrence of Acute myocardial infaction during follow-up period 


\begin{tabular}{|c|c|c|}
\hline 23-24.9 & $\begin{array}{l}0.926 \\
(0.813- \\
1.053)\end{array}$ & $\begin{array}{l}0.883(0.776- \\
1.005)\end{array}$ \\
\hline $25-29.9$ & $\begin{array}{l}1.008 \\
(0.89- \\
1.142)\end{array}$ & $\begin{array}{l}0.929(0.82- \\
1.052)\end{array}$ \\
\hline$\geq 30$ & $\begin{array}{l}1.34 \\
(1.066- \\
1.686)\end{array}$ & $\begin{array}{l}1.223(0.972- \\
1.54)\end{array}$ \\
\hline \multicolumn{3}{|l|}{ Hemoglobin (g/dL) } \\
\hline $\begin{array}{l}<13.5(\text { Male) },< \\
12(\text { Female) }\end{array}$ & $\begin{array}{l}1.498 \\
(1.273- \\
1.763)\end{array}$ & $\begin{array}{l}1.553(1.319- \\
1.829)\end{array}$ \\
\hline $\begin{array}{l}\text { 13.5-17.5(Male), } \\
12-15.5 \text { (Female) }\end{array}$ & 1 & 1 \\
\hline $\begin{array}{l}>17.5 \text { (Male), }> \\
15.5 \text { (Female) }\end{array}$ & $\begin{array}{l}1.446 \\
(1.047- \\
1.999)\end{array}$ & $\begin{array}{l}1.081(0.782- \\
1.494)\end{array}$ \\
\hline \multicolumn{3}{|l|}{ Creatinine (mg/dL) } \\
\hline$\leq 1.5$ & 1 & 1 \\
\hline$>1.5$ & $\begin{array}{l}1.735 \\
(1.455- \\
2.068)\end{array}$ & $\begin{array}{l}1.624(1.362- \\
1.937)\end{array}$ \\
\hline \multicolumn{3}{|l|}{$\begin{array}{l}\text { Total cholesterol } \\
(\mathrm{mg} / \mathrm{dL})\end{array}$} \\
\hline$<200$ & 1 & 1 \\
\hline $200-239$ & $\begin{array}{l}1.066 \\
(0.96- \\
1.184)\end{array}$ & $\begin{array}{l}0.888(0.799- \\
0.986)\end{array}$ \\
\hline$>239$ & $\begin{array}{l}1.606 \\
(1.417- \\
1.821)\end{array}$ & $\begin{array}{l}0.974(0.857- \\
1.107)\end{array}$ \\
\hline \multicolumn{3}{|l|}{ ALT (IU/L) } \\
\hline$<40$ & 1 & 1 \\
\hline
\end{tabular}

Abbreviation: Pre-MetS, pre-metabolic syndrome; MetS, metabolic syndrome, HR, hazard ratio *Occurrence of Acute myocardial infaction during follow-up period 


\begin{tabular}{|c|c|c|c|}
\hline & $\begin{array}{l}\text { Non-Adjusted } \\
\text { HR (95\% Cl) }\end{array}$ & Multivariable HR $(95 \% \mathrm{Cl})$ & \\
\hline $40-99$ & & $\begin{array}{l}1.092 \\
(0.973- \\
1.226)\end{array}$ & $\begin{array}{l}1.082(0.964- \\
1.214)\end{array}$ \\
\hline$>100$ & & $\begin{array}{l}1.436 \\
(1.067- \\
1.933)\end{array}$ & $\begin{array}{l}1.591(1.183- \\
2.139)\end{array}$ \\
\hline \multicolumn{4}{|c|}{$\begin{array}{l}\text { Acute myocardial } \\
\text { infarction* }\end{array}$} \\
\hline \multicolumn{3}{|l|}{ No } & 1 \\
\hline \multicolumn{3}{|l|}{ Yes } & $\begin{array}{l}130.854 \\
(118.389- \\
144.631)\end{array}$ \\
\hline \multicolumn{4}{|c|}{ Abbreviation: Pre-MetS, pre-metabolic syndrome; MetS, metabolic syndrome, HR, hazard ratio } \\
\hline \multicolumn{4}{|c|}{ *Occurrence of Acute myocardial infaction during follow-up period } \\
\hline
\end{tabular}


Table 4

Risk of heart failure in female: Cox proportional hazard model.

\begin{tabular}{|c|c|c|c|c|c|}
\hline \multirow[b]{2}{*}{ Metabolic status } & \multirow{2}{*}{$\begin{array}{l}\text { Non-Adjusted } \\
\text { HR ( } 95 \% \mathrm{Cl}) \\
\text { Model } 1\end{array}$} & \multicolumn{4}{|c|}{ Multivariable HR (95\% Cl) } \\
\hline & & Model 2 & Model 3 & Model 4 & Model 5 \\
\hline Normal & 1 & 1 & 1 & 1 & 1 \\
\hline Pre-metabolic synd. & $\begin{array}{l}1.605(1.34- \\
1.923)\end{array}$ & $\begin{array}{l}1.555 \\
(1.298- \\
1.864)\end{array}$ & $\begin{array}{l}1.548 \\
(1.291- \\
1.856)\end{array}$ & $\begin{array}{l}1.436 \\
(1.193- \\
1.73)\end{array}$ & $\begin{array}{l}1.395(1.158- \\
1.681)\end{array}$ \\
\hline Metabolic synd. & $\begin{array}{l}3.349(2.706- \\
4.145)\end{array}$ & $\begin{array}{l}3.118 \\
(2.515- \\
3.866)\end{array}$ & $\begin{array}{l}3.086 \\
(2.486- \\
3.83)\end{array}$ & $\begin{array}{l}2.324 \\
(1.817- \\
2.973)\end{array}$ & $\begin{array}{l}2.144(1.674- \\
2.747)\end{array}$ \\
\hline Age & & $\begin{array}{l}1.056 \\
(1.029- \\
1.084)\end{array}$ & $\begin{array}{l}1.056 \\
(1.029- \\
1.085)\end{array}$ & $\begin{array}{l}1.057 \\
(1.029- \\
1.085)\end{array}$ & $\begin{array}{l}1.051(1.023- \\
1.079)\end{array}$ \\
\hline \multicolumn{6}{|l|}{ Smoking status } \\
\hline Non-smokiner & & 1 & 1 & 1 & 1 \\
\hline Ex-smoker & & $\begin{array}{l}0.839 \\
(0.434- \\
1.624)\end{array}$ & $\begin{array}{l}0.838 \\
(0.433- \\
1.622)\end{array}$ & $\begin{array}{l}0.84 \\
(0.434- \\
1.626)\end{array}$ & $\begin{array}{l}0.847(0.437- \\
1.639)\end{array}$ \\
\hline Current smoker & & $\begin{array}{l}1.99 \\
(1.452- \\
2.726)\end{array}$ & $\begin{array}{l}1.99 \\
(1.452- \\
2.727)\end{array}$ & $\begin{array}{l}1.995 \\
(1.455- \\
2.735)\end{array}$ & $\begin{array}{l}1.721(1.254- \\
2.362)\end{array}$ \\
\hline \multicolumn{6}{|l|}{$\begin{array}{l}\text { Alcohol } \\
\text { consumption }\end{array}$} \\
\hline No drink & & & & & 1 \\
\hline 2-3 per month & & $\begin{array}{l}0.942 \\
(0.787- \\
1.127)\end{array}$ & $\begin{array}{l}0.942 \\
(0.787- \\
1.127)\end{array}$ & $\begin{array}{l}0.952 \\
(0.795- \\
1.14)\end{array}$ & $\begin{array}{l}0.964(0.805- \\
1.154)\end{array}$ \\
\hline 1-4 per week & & $\begin{array}{l}0.909 \\
(0.603- \\
1.37)\end{array}$ & $\begin{array}{l}0.909 \\
(0.603- \\
1.37)\end{array}$ & $\begin{array}{l}0.924 \\
(0.613- \\
1.392)\end{array}$ & $\begin{array}{l}0.931(0.618- \\
1.403)\end{array}$ \\
\hline$\geq$ per week & & $\begin{array}{l}1.353 \\
(0.787- \\
2.324)\end{array}$ & $\begin{array}{l}1.354 \\
(0.788- \\
2.326)\end{array}$ & $\begin{array}{l}1.363 \\
(0.793- \\
2.341)\end{array}$ & $\begin{array}{l}1.435(0.837- \\
2.461)\end{array}$ \\
\hline \multicolumn{6}{|l|}{ Exercise } \\
\hline No exercise & & 1 & 1 & 1 & 1 \\
\hline
\end{tabular}

Abbreviation: Pre-MetS, pre-metabolic syndrome; MetS, metabolic syndrome, HR, hazard ratio *Occurrence of Acute myocardial infaction during follow-up period 


\begin{tabular}{|c|c|c|c|c|c|}
\hline \multirow[b]{2}{*}{ 1-4 per week } & \multirow{2}{*}{$\begin{array}{l}\text { Non-Adjusted } \\
\text { HR (95\% Cl) }\end{array}$} & \multicolumn{4}{|c|}{ Multivariable HR ( $95 \% \mathrm{Cl})$} \\
\hline & & $\begin{array}{l}0.941 \\
(0.769- \\
1.151)\end{array}$ & $\begin{array}{l}0.938 \\
(0.767- \\
1.148)\end{array}$ & $\begin{array}{l}0.939 \\
(0.767- \\
1.149)\end{array}$ & $\begin{array}{l}0.943(0.771- \\
1.155)\end{array}$ \\
\hline$\geq 5$ per week & & $\begin{array}{l}0.898 \\
(0.755- \\
1.066)\end{array}$ & $\begin{array}{l}0.894 \\
(0.752- \\
1.063)\end{array}$ & $\begin{array}{l}0.901 \\
(0.758- \\
1.07)\end{array}$ & $\begin{array}{l}0.915(0.77- \\
1.088)\end{array}$ \\
\hline \multicolumn{6}{|c|}{$\begin{array}{l}\text { Family history of } \\
\text { heart disease }\end{array}$} \\
\hline No & & & 1 & 1 & 1 \\
\hline Yes & & & $\begin{array}{l}1.08 \\
(0.792- \\
1.474)\end{array}$ & $\begin{array}{l}1.088 \\
(0.797- \\
1.484)\end{array}$ & $\begin{array}{l}1.088(0.798- \\
1.485)\end{array}$ \\
\hline \multicolumn{6}{|c|}{$\begin{array}{l}\text { Family history of } \\
\text { hypertension }\end{array}$} \\
\hline No & & & 1 & 1 & 1 \\
\hline Yes & & & $\begin{array}{l}1.13 \\
(0.942- \\
1.356)\end{array}$ & $\begin{array}{l}1.126 \\
(0.939- \\
1.351)\end{array}$ & $\begin{array}{l}1.086(0.905- \\
1.303)\end{array}$ \\
\hline \multicolumn{6}{|c|}{$\begin{array}{l}\text { Family history of } \\
\text { diabetes mellitus }\end{array}$} \\
\hline No & & & 1 & 1 & 1 \\
\hline Yes & & & $\begin{array}{l}0.976 \\
(0.795- \\
1.197)\end{array}$ & $\begin{array}{l}0.972 \\
(0.792- \\
1.193)\end{array}$ & $\begin{array}{l}0.985(0.802- \\
1.209)\end{array}$ \\
\hline \multicolumn{6}{|c|}{$\begin{array}{l}\text { Family history of } \\
\text { stroke }\end{array}$} \\
\hline No & & & 1 & 1 & 1 \\
\hline Yes & & & $\begin{array}{l}0.966 \\
(0.753- \\
1.238)\end{array}$ & $\begin{array}{l}0.972 \\
(0.758- \\
1.246)\end{array}$ & $\begin{array}{l}0.974(0.76- \\
1.249)\end{array}$ \\
\hline \multicolumn{6}{|c|}{ Body mass index } \\
\hline$<18.5$ & & & & $\begin{array}{l}1.647 \\
(1.043-2.6)\end{array}$ & $\begin{array}{l}1.663(1.053- \\
2.624)\end{array}$ \\
\hline $18.5-22.9$ & & & & 1 & 1 \\
\hline
\end{tabular}

Abbreviation: Pre-MetS, pre-metabolic syndrome; MetS, metabolic syndrome, HR, hazard ratio *Occurrence of Acute myocardial infaction during follow-up period 


\begin{tabular}{|c|c|c|c|c|}
\hline & $\begin{array}{l}\text { Non-Adjusted } \\
\text { HR (95\% Cl) }\end{array}$ & Multivariable HR (95\% Cl) & & \\
\hline 23-24.9 & & & $\begin{array}{l}1.233 \\
(1.014-1.5)\end{array}$ & $\begin{array}{l}1.226(1.008- \\
1.492)\end{array}$ \\
\hline 25-29.9 & & & $\begin{array}{l}1.305 \\
(1.07- \\
1.593)\end{array}$ & $\begin{array}{l}1.273(1.042- \\
1.554)\end{array}$ \\
\hline$>=30$ & & & $\begin{array}{l}2.152 \\
(1.571- \\
2.946)\end{array}$ & $\begin{array}{l}2.085(1.523- \\
2.853)\end{array}$ \\
\hline \multicolumn{5}{|l|}{ Hemoglobin $(g / d L)$} \\
\hline $\begin{array}{l}<13.5(\text { Male) },< \\
\text { 12(Female) }\end{array}$ & & & $\begin{array}{l}1.207 \\
(1.009- \\
1.444)\end{array}$ & $\begin{array}{l}1.203(1.006- \\
1.439)\end{array}$ \\
\hline $\begin{array}{l}\text { 13.5-17.5(Male), } \\
\text { 12-15.5 (Female) }\end{array}$ & & & 1 & 1 \\
\hline $\begin{array}{l}>17.5(\text { Male }),> \\
15.5(\text { Female })\end{array}$ & & & $\begin{array}{l}1.562 \\
(0.775- \\
3.148)\end{array}$ & $\begin{array}{l}1.496(0.742- \\
3.015)\end{array}$ \\
\hline \multicolumn{5}{|l|}{ Creatinine $(\mathrm{mg} / \mathrm{dL})$} \\
\hline$\leq 1.5$ & & & 1 & 1 \\
\hline$>1.5$ & & & $\begin{array}{l}2.3(1.603- \\
3.298)\end{array}$ & $\begin{array}{l}2.167(1.51- \\
3.109)\end{array}$ \\
\hline \multicolumn{5}{|l|}{$\begin{array}{l}\text { Total cholesterol } \\
\text { (mg/dL) }\end{array}$} \\
\hline$<200$ & & & 1 & 1 \\
\hline $200-239$ & & & $\begin{array}{l}0.966 \\
(0.818- \\
1.14)\end{array}$ & $\begin{array}{l}0.977(0.827- \\
1.153)\end{array}$ \\
\hline$>239$ & & & $\begin{array}{l}1.038 \\
(0.818- \\
1.318)\end{array}$ & $\begin{array}{l}1.011(0.797- \\
1.283)\end{array}$ \\
\hline \multicolumn{5}{|l|}{ ALT (IU/L) } \\
\hline$<40$ & & & 1 & 1 \\
\hline 40-99 & & & $\begin{array}{l}1.643 \\
(1.265- \\
2.132)\end{array}$ & $\begin{array}{l}1.551(1.193- \\
2.016)\end{array}$ \\
\hline \multicolumn{5}{|c|}{ Abbreviation: Pre-MetS, pre-metabolic syndrome; MetS, metabolic syndrome, HR, hazard ratio } \\
\hline
\end{tabular}




\begin{tabular}{|lcc|}
\hline \multicolumn{2}{|c|}{$\begin{array}{c}\text { Non-Adjusted } \\
\text { HR (95\% Cl) }\end{array}$} & Multivariable HR (95\% Cl) \\
\hline$>100$ & 1.942 & $1.753(0.869-$ \\
& $(0.963-$ & $3.537)$ \\
\hline $\begin{array}{l}\text { Acute myocardial } \\
\text { infarction* }\end{array}$ & $3.914)$ & \\
\hline No & 1 \\
\hline Yes & 82.637 \\
& & $(61.643-$ \\
\hline Abbreviation: Pre-MetS, pre-metabolic syndrome; MetS, metabolic syndrome, HR, hazard ratio \\
\hline *Occurrence of Acute myocardial infaction during follow-up period & \\
\hline
\end{tabular}

In addition, the occurrence of AMI during follow-up periodwas associated with a 131-fold and 83-fold higher risk for HF in men and women, respectively. Although the study participants were all in their fifties, the risk of $\mathrm{HF}$ increased with increasing age. Current smoking increased the risk of HF by 1.2-fold (HR (95\% Cl): 1.19 (1.051-1.348)) in men and 1.7-fold in women (HR (95\% Cl): 1.721 (1.254-2.362)). Low hemoglobin levels and elevated creatinine levels were also risk factors for HF. The HR $(95 \% \mathrm{Cl})$ of low hemoglobin was 1.553 (1.319-1.829) in men and 1.203 (1.006-1.439) in women; the $\mathrm{HR}(95 \% \mathrm{Cl})$ of elevated creatinine level was 1.624 (1.362-1.937) in men and 2.167 (1.51-3.109) in women.

$\mathrm{BMI}$ as a risk factor for HF showed different results by sex. Overweight was a significant risk factor for HF in women with obesity $\left(\mathrm{BMI} \geq 30 \mathrm{~kg} / \mathrm{m}^{2}\right)$, increasing the risk by 2-fold ( $\mathrm{HR}(95 \% \mathrm{Cl}): 2.085$ (1.5232.853)), whereas overweight was not a risk factor for $\mathrm{HF}$ in men. Underweight (BMI $\left.<18.5 \mathrm{~kg} / \mathrm{m}^{2}\right)$ increased the risk of HF in both men and women by 1.8 -fold $(\mathrm{HR}(95 \% \mathrm{Cl}): 1.786(1.266-2.52))$ and 1.7fold (HR (95\% Cl): $1.663(1.053-2.624))$, respectively.

Alcohol consumption, total cholesterol and ALT level exhibited inconsistent relationships with the occurrence of HF by sex. Only in men, the consumption of 2-3 servings of alcohol per month and slight elevation of total cholesterol (200-239 (mg/dL)) decreased the risk of HF by $19 \%$ (HR (95\% Cl): 0.814 (0.729-0.909)) and $11 \%(\mathrm{HR}(95 \% \mathrm{Cl}): 0.888(0.799-0.986)$, respectively). Slight elevation of the ALT level (40-99 (IU/L)) increased the risk of HF (HR (95\% Cl): 1.551 (1.193-2.016)) only in women, and an elevation of the ALT level over 100 (IU/L) increased the risk of HF (HR (95\% Cl): 1.591 (1.183-2.139)) only in men.

\section{Discussion}

The associations between the prevalence or prognosis of HF and MetS have been shown in many studies. ${ }^{8-11}$ However, most of these studies were cross-sectional in design, which prevents drawing 
causal inferences, and only a few longitudinal studies have been reported. A longitudinal study with 2314 middle-aged men reported that MetS was a significant risk factor for $\mathrm{HF}$, with an $\mathrm{HR}(95 \% \mathrm{Cl})$ of 1.80 (1.11 to 2.91). ${ }^{16} \mathrm{~A}$ study of elderly individuals in their seventies reported that MetS increased the occurrence of $\mathrm{HF}$, with an $\mathrm{HR}(95 \% \mathrm{Cl})$ of $1.49(1.10-2.00),{ }^{17}$ and another study of elderly individuals with a mean age of 69 years also reported that MetS was a predictor of HF with an HR $(95 \% \mathrm{Cl})$ of $1.58(1.16-2.15) .{ }^{18} \mathrm{In}$ contrast, in a study of participants with a mean age of 62 years old, MetS was not a significant risk factor for $\mathrm{HF}^{13}$ The present study was a large population-based study that included over 2 million individuals in their fifties. MetS was a significant risk factor for HF, and the HR in men was similar to that reported in a study of middle-aged men. ${ }^{16}$ Although the results of studies in elderly populations were inconsistent, the HR in the present study was higher than that in a study conducted with elderly participants in which MetS was reported to be a significant risk factor for $\mathrm{HF}^{17,18}$

The present study revealed that the effect of MetS on HF differs by sex. The HR $(95 \% \mathrm{Cl})$ in men was 1.711 (1.433-2.044) and that in women was 2.144 (1.674-2.747), showing a higher risk of MetS associated with HF in women than in men. To the best of our knowledge, this is the first study to demonstrate the sex difference of MetS as a risk factor for HF.

In this study, we considered the known risk factors for HF as covariates for adjustment in the Cox regression analysis. It is well known that $\mathrm{AMI}$ is a major risk factor for HF. In the study with a median follow-up time of 3.2 years, $31 \%$ of men and $46 \%$ of women developed HF among those hospitalized due to AMI. ${ }^{19}$ Another study reported that approximately $84 \%$ of patients with coronary heart disease developed HF during the 19-year follow-up. ${ }^{20}$ In the present study, AMI increased the risk of HF by 131 fold in men and 83-fold in women, which are considerably higher risks than those previously reported. In the National Health and Nutrition Examination Survey (NHANES) study, ${ }^{20}$ the relative risk of AMI was 8 , and in the Framingham study, ${ }^{21}$ the HR was 6 . The reason that the HR of AMI was extremely high in our study was thought to be that as the study population was composed of relatively healthy individuals and patients with a history of cardiocerebral vascular diseases were excluded; additionally, the follow-up period was relatively short compared to that of the NHANES study or Framingham study. Namely, in subjects who do not have underlying disease that can lead to HF, the effect of AMI on the occurrence of HF within a short period is suspected to be critical. The HR of AMI for HF was higher in men than in women, which coincided with the findings of a previous report. ${ }^{20}$

The association between BMI and HF exhibited obvious sex differences. In women, BMI of 23.0-29.9 $\mathrm{kg} / \mathrm{m}^{2}$ increased the risk of HF by 1.2 - to 1.3 -fold, and obesity (BMI $\geq 30 \mathrm{~kg} / \mathrm{m}^{2}$ ) increased it by 2.1 -fold, whereas in men, overweight was not a significant risk factor for HF. This result is consistent with many studies that reported that overweight increased the HF risk in women more significantly than in men. ${ }^{20 \text {, }}$ 22-24 In the present study, underweight $\left(\mathrm{BMI}<18.5 \mathrm{~kg} / \mathrm{m}^{2}\right)$ increased the risk of HF in both men and women. Although various studies reported the association between BMI and HF, underweight has rarely been separately evaluated. ${ }^{25-27}$ The increased risk of HF by underweight is supported by a recent study that reported associations among obesity degree, glycemic status, and risk of HF. ${ }^{28}$ 
In this study, low hemoglobin was a risk factor for HF; however, high hemoglobin levels were not a risk factor despite the significantly high prevalence of HF in the high hemoglobin level population. Low hemoglobin levels and lower blood viscosity, hypoxia and enhanced nitric oxide (NO) activity induce reduced vascular resistance, followed by increased cardiac output. Increased cardiac output leads to left ventricular hypertrophy and cardiac enlargement, which can eventually lead to HF. ${ }^{29}$ Different results have been reported regarding the association between $\mathrm{HF}$ and hemoglobin level; while Klip et al. ${ }^{30}$ reported that both low levels and high levels of hemoglobin increased the risk of HF compared to the risk associated with normal hemoglobin levels, Coglianese et al. ${ }^{31}$ reported that high and normal hematocrit levels were associated with a higher risk of HF than low hematocrit levels. These reports explained that a high level of hemoglobin increases vascular resistance by scavenging nitric oxide, a vasodilator, with induces hypertension, left ventricular hypertrophy and finally HF. These different associations between hemoglobin level and HF risk are suspected to result from the different follow-up periods and different study populations. While the follow-up period of our study was relatively short, with a maximum of 8 years, the study of hematocrit and $\mathrm{HF}^{31}$ was performed over 20 years, and the study of hemoglobin and HF had a follow-up of a median of 6 years. ${ }^{30}$ Additionally, while our study was performed in a healthy population without underlying cardiovascular disease, which is closely related to HF, the study of hemoglobin and $\mathrm{HF}^{30}$ did not exclude the population with those underlying diseases.

Despite the positive aspects of the present study, there were some limitations that should be addressed. First, this study evaluated the subjects' metabolic status based on data from 2009. Even if a subject who had been classified into the normal group on the basis of the data from 2009 developed MetS during the follow-up period, the subject was analyzed in the normal group. Although the number of subjects whose metabolic status was changed may not be substantial considering the total study population, there can be an effect on the accuracy of the results. Second, our study excluded subjects with a history of malignancy or cardiocerebral vascular disease; thus, the association between MetS status and HF risk was analyzed in a relatively healthy population, which means that this result may not be generalizable to high-risk populations.

\section{Conclusion}

In summary, this study evaluated the association between MetS and the risk of HF in middle-aged men and women using nationwide real-world data. MetS was a risk factor for HF, and its effect on HF was stronger in women than in men. Not only MetS but also pre-MetS was a predictor of HF, but pre-MetS was associated with a lower risk than MetS.

\section{Declarations}

- Ethics approval and consent to participate

Institutional Review Board of Konkuk University Medical Center (No. KUH 2020-07-096) 
- Consent for publication

Not applicable

- Availability of data and materials

The data that support the findings of this study are available from National Health Insurance Service (NHIS) but restrictions apply to the availability of these data, which were used under license for the current study, and so are not publicly available. Data are however available from the authors upon reasonable request and with permission of NHIS.

- Competing interests

The authors declare that they have no competing interests

- Funding

There is no funding source in this study.

- Authors' contributions

Tae-Eun Kim: analysis and interpretation of data; drafting and revision of manuscript

Hyeongsu Kim: design of the work; analysis and interpretation of data; revision of manuscript

JiDong Sung: interpretation of data; revision of manuscript

Duk-Kyung Kim: interpretation of data; revision of manuscript

Myoung-Soon Lee: interpretation of data; revision of manuscript

Seong Woo Han: interpretation of data; revision of manuscript

Hyun-Joong Kim: interpretation of data; revision of manuscript

Sung Hea Kim: conception and design of the study; interpretation of data; revision of the manuscript Kyu-Hyung Ryu: conception and design of the study; interpretation of data; revision of the manuscript

- Acknowledgements

Not applicable

\section{References}

1. Coronel R, de Groot JR, van Lieshout JJ. Defining heart failure. Cardiovasc Res. 2001;50:419-22. 
2. Tan LB, Williams SG, Tan DK, Cohen-Solal A. So many definitions of heart failure: are they all universally valid? A critical appraisal. Expert Rev Cardiovasc Ther. 2010;8:217-28.

3. Braunwald E. Heart failure. JACC Heart Fail. 2013;1:1-20.

4. Askoxylakis V, Thieke C, Pleger ST, Most P, Tanner J, Lindel K, Katus HA, Debus J, Bischof M. Longterm survival of cancer patients compared to heart failure and stroke: a systematic review. BMC Cancer. 2010;10:105.

5. Aguilar M, Bhuket T, Torres S, Liu B, Wong RJ. Prevalence of the metabolic syndrome in the United States, 2003-2012. JAMA. 2015;313:1973-4.

6. Gao W. DECODE Study Group. Does the constellation of risk factors with and without abdominal adiposity associate with different cardiovascular mortality risk? Int J Obes (Lond). 2008;32:757-62.

7. Rochlani Y, Pothineni NV, Kovelamudi S, Mehta JL. Metabolic syndrome: pathophysiology, management, and modulation by natural compounds. Ther Adv Cardiovasc Dis. 2017;11:215-25.

8. Miura Y, Fukumoto Y, Shiba N, Miura T, Shimada K, Iwama Y, Takagi A, Matsusaka H, Tsutsumi T, Yamada A, Kinugawa S, Asakura M, Okamatsu S, Tsutsui H, Daida H, Matsuzaki M, Tomoike H, Shimokawa H. Prevalence and clinical implication of metabolic syndrome in chronic heart failure. Circ J. 2010;74:2612-21.

9. Li C, Ford ES, McGuire LC, Mokdad AH. Association of metabolic syndrome and insulin resistance with congestive heart failure: findings from the Third National Health and Nutrition Examination Survey. J Epidemiol Community Health. 2007;61:67-73.

10. Tamariz L, Hassan B, Palacio A, Arcement L, Horswell R, Hebert K. Metabolic syndrome increases mortality in heart failure. Clin Cardiol. 2009;32:327-31.

11. Perrone-Filardi P, Savarese G, Scarano M, Cavazzina R, Trimarco B, Minneci S, Maggioni AP, Tavazzi L, Tognoni G, Marchioli R. Prognostic impact of metabolic syndrome in patients with chronic heart failure: data from GISSI-HF trial. Int J Cardiol. 2015;178:85-90.

12. Koutroumpakis E, Jozwik B, Aguilar D, Taegtmeyer H. Strategies of Unloading the Failing Heart from Metabolic Stress. Am J Med. 2020;133:290-6.

13. Bahrami H, Bluemke DA, Kronmal R, Bertoni AG, Lloyd-Jones DM, Shahar E, Szklo M, Lima JA. Novel metabolic risk factors for incident heart failure and their relationship with obesity: the MESA (MultiEthnic Study of Atherosclerosis) study. J Am Coll Cardiol. 2008;51:1775-83.

14. Redfield MM, Jacobsen SJ, Burnett JC Jr, Mahoney DW, Bailey KR, Rodeheffer RJ. Burden of systolic and diastolic ventricular dysfunction in the community: appreciating the scope of the heart failure epidemic. JAMA. 2003;289:194-202.

15. Lakatta EG. Arterial and cardiac aging: major shareholders in cardiovascular disease enterprises: Part III: cellular and molecular clues to heart and arterial aging. Circulation. 2003;107:490-7.

16. Ingelsson E, Arnlov J, Lind L, Sundstrom J. Metabolic syndrome and risk for heart failure in middleaged men. Heart. 2006;92:1409-13. 
17. Butler J, Rodondi N, Zhu Y, Figaro K, Fazio S, Vaughan DE, Satterfield S, Newman AB, Goodpaster B, Bauer DC, Holvoet P, Harris TB, de Rekeneire N, Rubin S, Ding J. Kritchevsky SB and Health ABC Study. Metabolic syndrome and the risk of cardiovascular disease in older adults. J Am Coll Cardiol. 2006;47:1595-602.

18. Wang J, Sarnola K, Ruotsalainen S, Moilanen L, Lepisto P, Laakso M, Kuusisto J. The metabolic syndrome predicts incident congestive heart failure: a 20-year follow-up study of elderly Finns. Atherosclerosis. 2010;210:237-42.

19. Sulo G, Igland J, Vollset SE, Nygard O, Ebbing M, Sulo E, Egeland GM, Tell GS. Heart failure complicating acute myocardial infarction; burden and timing of occurrence: a nation-wide analysis including 86771 patients from the cardiovascular disease in Norway (CVDNOR) project. J Am Heart Assoc. 2016;5:e002667.

20. He J, Ogden LG, Bazzano LA, Vupputuri S, Loria C, Whelton PK. Risk factors for congestive heart failure in US men and women: NHANES I epidemiologic follow-up study. Arch Intern Med. 2001;161:996-1002.

21. Levy D, Larson MG, Vasan RS, Kannel WB, Ho KK. The progression from hypertension to congestive heart failure. JAMA. 1996;275:1557-62.

22. Kenchaiah S, Evans JC, Levy D, Wilson PW, Benjamin EJ, Larson MG, Kannel WB, Vasan RS. Obesity and the risk of heart failure. N Engl J Med. 2002;347:305-13.

23. Savji N, Meijers WC, Bartz TM, Bhambhani V, Cushman M, Nayor M, Kizer JR, Sarma A, Blaha MJ, Gansevoort RT, Gardin JM, Hillege HL, Ji F, Kop WJ, Lau ES, Lee DS, Sadreyev R, van Gilst WH, Wang TJ, Zanni MV, Vasan RS, Allen NB, Psaty BM, van der Harst P, Levy D, Larson M, Shah SJ, de Boer RA. Gottdiener JS and Ho JE. The Association of Obesity and Cardiometabolic Traits With Incident HFpEF and HFrEF. JACC Heart Fail. 2018;6:701-9.

24. Eaton CB, Pettinger M, Rossouw J, Martin LW, Foraker R, Quddus A, Liu S, Wampler NS, Hank Wu WC, Manson JE, Margolis K, Johnson KC, Allison M, Corbie-Smith G, Rosamond W. Breathett K and Klein L. Risk factors for incident hospitalized heart failure with preserved versus reduced ejection fraction in a multiracial cohort of postmenopausal women. Circ Heart Fail. 2016;9:e002883.

25. Huffman MD, Berry JD, Ning H, Dyer AR, Garside DB, Cai X, Daviglus ML, Lloyd-Jones DM. Lifetime risk for heart failure among white and black Americans: cardiovascular lifetime risk pooling project. J Am Coll Cardiol. 2013;61:1510-7.

26. Bjorck L, Lundberg $C$, Schaufelberger M, Lissner L, Adiels M, Rosengren A. Body mass index in women aged 18 to 45 and subsequent risk of heart failure. Eur J Prev Cardiol. 2020;27:1165-74.

27. Rosengren A, Aberg M, Robertson J, Waern M, Schaufelberger M, Kuhn G, Aberg D, Schioler L, Toren $\mathrm{K}$. Body weight in adolescence and long-term risk of early heart failure in adulthood among men in Sweden. Eur Heart J. 2017;38:1926-33.

28. Rhee EJ, Kwon H, Park SE, Han KD, Park YG, Kim YH, Lee WY. Associations among obesity degree, glycemic status, and risk of heart failure in 9,720,220 Korean adults. Diabetes Metab J. 2020;44:592-601. 
29. Varat MA, Adolph RJ, Fowler NO. Cardiovascular effects of anemia. Am Heart J. 1972;83:415-26.

30. Klip IT, Postmus D, Voors AA, Brouwers FP, Gansevoort RT, Bakker SJ, Hillege HL, de Boer RA, van der Harst P, van Gilst WH, van Veldhuisen DJ, van der Meer P. Hemoglobin levels and new-onset heart failure in the community. Am Heart J. 2015;169:94-101.e2.

31. Coglianese EE, Qureshi MM, Vasan RS, Wang TJ, Moore LL. Usefulness of the blood hematocrit level to predict development of heart failure in a community. Am J Cardiol. 2012;109:241-5.

\section{Figures}

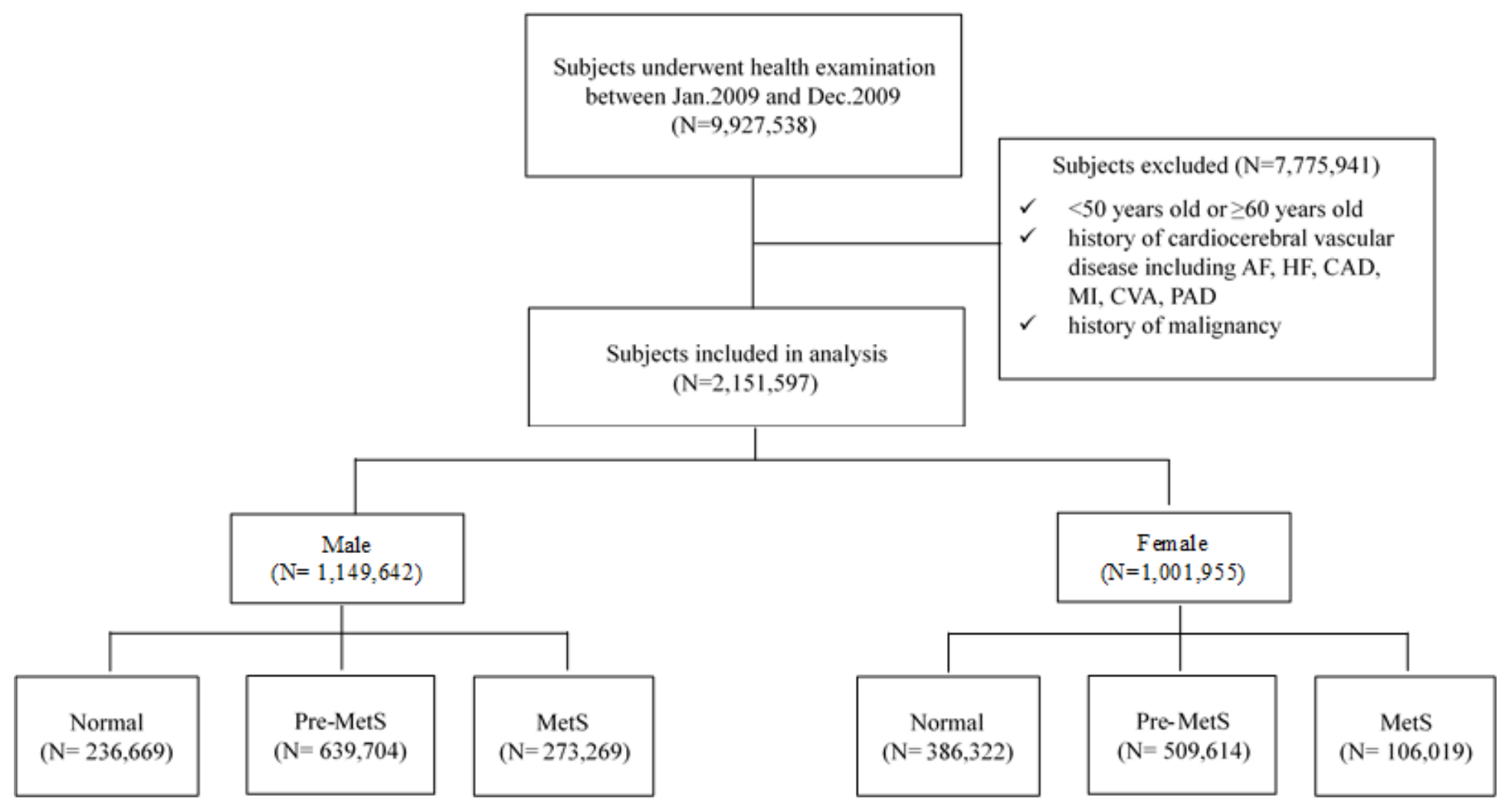

\section{Figure 1}

Flow diagram of study population. Abbreviation: Pre-MetS, pre-metabolic syndrome; MetS, metabolic syndrome; $\mathrm{AF}$, atrial fibrillation; $\mathrm{HF}$, heart failure; $\mathrm{CAD}$, coronary artery disease; $\mathrm{MI}$, myocardial infarction; CVA, cerebrovascular accident; $P A D$, peripheral arterial disease 


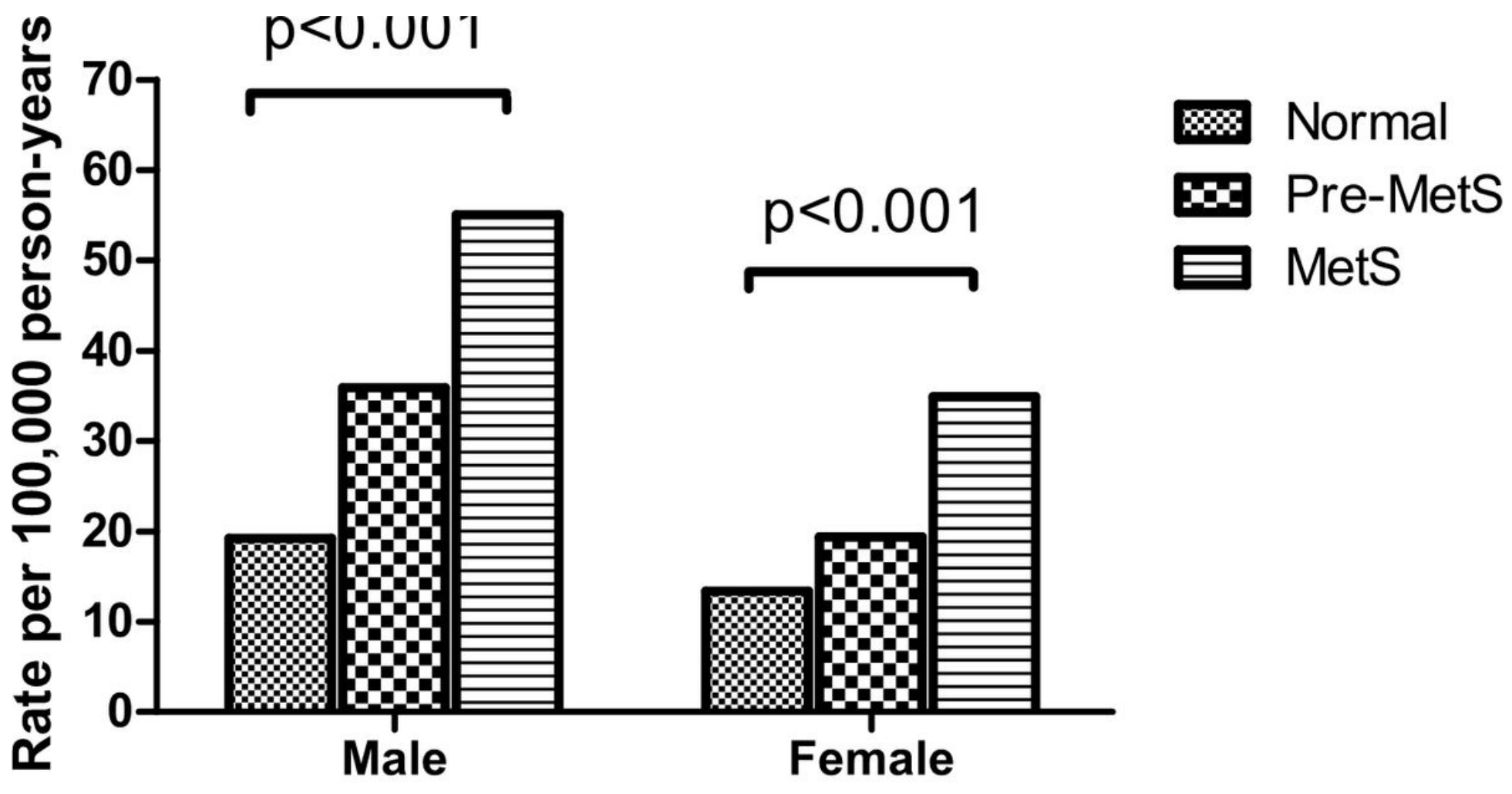

Figure 2

Incidence rate of heart failure (Abbreviation: Pre-MetS, pre-metabolic syndrome; MetS, metabolic syndrome) 